\title{
Un amphipode stygobie de la Thaïlande (Crustacea : Amphipoda)
}

\author{
L. Botosaneanu' \\ J. Notenboom 1
}

Mots-clés : Amphipoda, faune stygobie, Thaïlande.

On décrit Bogidiella (Bogidiella) thai sp. n. d'une grotte de Thaîlande méridionale. Il s'agit du premier amphipode stygobie découvert dans les eaux continentales, douces, de la Péninsule Indochinoise.

\section{A st ygobiont amphipod from Thailand (Crustacea : Amphipoda}

Keywords : Amphipoda, stygobiont fauna, Thailand.

Bogidiella (Bogidiella) thai sp. $\mathrm{n}$. is described from a cave in South Thailand. This is the first stygobiont amphipod discovered in the continental fresh-water of the Indochinese Peninsula.

Lors d'une de leurs expéditions en Asie du SudEst (Expédition Thai-Maros 86 organisée par l'As. sociation Pyrénéenne de Spéléologie), les membres d'un groupe de biospéléologues de l'Université de Toulouse dirigé par le $\mathrm{D} r \mathrm{~L}$. Deharveng, ont capturé dans une grotte de Thaïlande le premier amphipode stygobie d'eau douce (continentale) de la Péninsule Indochinoise. Il s'agit d'une espèce nouvelle de Bogidiella Hertzog, 1933, que nous décrivons.

\section{Bogidiella (Bogidiella) thai sp. $n$.}

Matériel et localité. - Tham (grotte) Poung Chang, en Thaïlande du Sud, Province de Phangnga, près de la ville du même nom. Le 12 juin 1986, 5, exemplaires collectés dans un petit bassin à fond limon'ux, dans un ruisselet affluent de la rivière souterraine principale, à environ $250 \mathrm{~m}$ de l'entrée de la grotte. Température de l'eau : $25,7-25,8^{\circ} \mathrm{C} ; \mathrm{pH}=$ 7,80-7,87. Leg. Véronique Bouguenec et Louis

1. Institut de Zoologie Taxonomique de l'Université d'Amsterdam, B.P. 20125, 1000 HC Amsterdam, Pays-Bas.
Deharveng. Les exemplaires sont passablement endommagés. Le matériel ( $\&$ holotype, $\sigma$ allotype, les deux partiellement disséqués, les appendices étant préparés dans la gomme de Faure sur plusieurs lames ; 3 paratypes non disséqués, en alcool) se trouve au Musée Zoologique de l'Université d'Amsterdam (coll. no. Amph. 108.239 a, b, c). Les figures ont été réalisées sur l'holotype $\odot$ et sur l'allotype $\sigma$.

Description. - L'holotype $q$ mesure $3,14 \mathrm{~mm}$; l'allotype ${ }^{\circ}$ est long de $2,89 \mathrm{~mm}$; les trois paratypes ont $2,78 \mathrm{~mm}, 2,45 \mathrm{~mm}$ et $2,15 \mathrm{~mm}$ respectivement.

Corps totalement dépigmenté ; pas de pigment oculaire. Aucun exemplaire ne porte des œufs. La $q$ holotype présente des oostégites allongés et glabres sur les péréionites 2.5 et des branchies coxales avec pédoncule allongé sur les péréionites 4-6 (fig. 1). Plaques coxales ( $f$ ig. 2 ); il y a un léger chevauchement des premières plaques, même quand l'animal est en extension; la largeur des plaques dépasse leur longueur maximum; la plaque 5 est la plus grande de toutes; une soie sur le bord des 
plaques $1,2,3,6$ et 7 , deux soies sur la plaque 4,3 soies sur la plaque 5. Plaques épimérales (fig. 3) à projection postéroventrale petite mais pointue, chaque plaque avec une sétule au bord postérieur, à implantation de plus en plus supérieure de la 1 ere à la $3^{\mathrm{e}}$ plaque.

Antenne 1 (Fig. 4). Dans l'exemplaire le plus grand, elle atteint environ $35 \%$ de la Iongueur totale du corps. Pédoncule dépassant en longueur le flagelle ; articles 1-3 du pédoncule de plus en plus courts. Flagelle de 8 segments (dans les exemplaires les plus petits : 6 ou 7); un seul aesthétasque - à très court pédoncule néanmoins bien visible - est présent sur chacun des segments 4-8 (dans les exemplaires les plus petits cette formule peut être $2-6$, ou $3,5,6,7$ ) ; chaque aesthétasque est approximativement aussi long que le segment suivant du flagelle, à l'exception de celui du segment apical, qui est beaucoup plus court. Flagelle accessoire bisegmenté, atteignant le milieu du $3^{e}$ segment du flagelle principal son $2^{\mathrm{e}}$ segment a seulement $1 / 4$ de la longueur du segment basal.

Antenne 2 (Fig. 5). Cône glandulaire bien développé. Flagelle de 5 segments, sa longueur à peu près égale à celle du dernier segment du pédoncule; un seul aesthétasque non pédonculé sur les segments 2 et 5.

\section{Labrum et labium: fig. 6-7.}

Mandibules (fig. 8-10). Près de la base du palpe, le corps mandibulaire présente un lobe inerme bien développé. Pars molaris fort développée : haute protubérance surmontée d'une couronne de fins denticules. Lacinia mobilis robuste; dans la mandibule gauche ( $\odot$ : fig. 8) elle est plus massive, à 5 assez for. tes dents; dans celle droite ( $Q$ : fig. 9) el le est apparemment plus plate, avec un grand nombre de minuscules dents. Entre lacinia mobilis et pars molaris s'insèrent 4 éléments qui ne peuvent pas être décrits comme soies pennées, car ce sont des éléments courts et aplatis, dans la mandibule gauche finement mais visiblement ciliés à l'extrémité, dans celle droite dépourvus ou pratiquement dépourvus de ciliation, et encore plus trapus. Dans la mandibule gauche du $\sigma$ lacinia mobilis se présente exactement comme chez la $Q$; dans la mandibule droite, nous l'avons observée (fig. 10) comme un simple ergot sans aucune denticulation, celle-ci étant peutêtre usée. Palpe mandibulaire : le $2^{e}$ segment est le plus long, et pourvu subapicalement sur le bord interne d'une soie ; segment distal à 2 soies distales ; une soie dans le tiers distal du bord interne a été observée chez le $\sigma$ seulement (fig. 10).

Maxille 1 (fig. 11-12). Sur l'apex du lobe interne, 3 soies pennées. A l'apex du lobe externe, 7 solides épines diversement denticulées à leur partie distale (fig. 12). Palpe à deux segments ; trois ou quatre soies pennées et pourvues d'une sétule secondaire basale, à l'apex du second segment.

Maxille 2 (fig. 13-14) avec les deux lobes fort allongés; 7 soies apicales ou subapicales sur le lobe interne, et 10 sur le lobe externe, ces dernières formant deux rangées.

Maxillipede (fig. 15-19). Les proportions des quatre segments du palpe sont bien représentées par les figures 15-16. Les deux lobes sont allongés. Une soie du côté médian du $1^{\text {er }}$ segment du palpe ; 6 soies, pennées ou non, formant une rangée près du bord interne du $2^{e}$ segment (la plus distale de ces soies peut être curieusement bifide); le $4^{\text {e }}$ segment se retrécit considérablement vers l'extrémité distale, où il se prolonge du côté externe par un « bec "bien distinct, surplombant la racine de la griffe gracile et longue : deux soies s'insèrent sur le bord interne du $4^{\mathrm{e}}$ segment du palpe, tout près de la racine de la griffe; il y a (fig. 16) une longue rangée de fins et longs cils sur une des faces du $4^{e}$ segment du palpe, et, du côté opposé, un groupe inportant de longs cils placés sur ce qui semble être une protubérance (mais celle-ci pourrait appartenir, au moins partiellement, au $3^{\text {e }}$ segment). Juste à l'apex du lobe externe (fig. 17) il y a 3 solides épines à extrémité fort obtuse, et trois soies en position subapicale. Remarquable est l'armature apicale du lobe interne (les fig. 18.19 représentent fidèlement les relations entre ces éléments). On y observe : 3 soies de longueur inégale, la plus longue implantée latéralement et non pennée, les deux autres pennées (la plus courte de ces soies est épaissie) ; ensuite, deux solides épines ressemblant à celles du lobe externe, mais dont l'apex peut être biparti ; enfin, près de l'angle interne deux curieux éléments qui sont de fortes épines garnies de nombreux longs cils.

Gnathopode 1 (fig. 20). Basis robuste, une longue soie sur son bord postérieur, deux soies fort courtes aux angles de son bord distal. Ischion avec une soie à l'angle postero-distal, où il y a aussi une zone de cils. Meros à deux soies distales et zone de cils. Carpe caractérisé par le très fort développement 


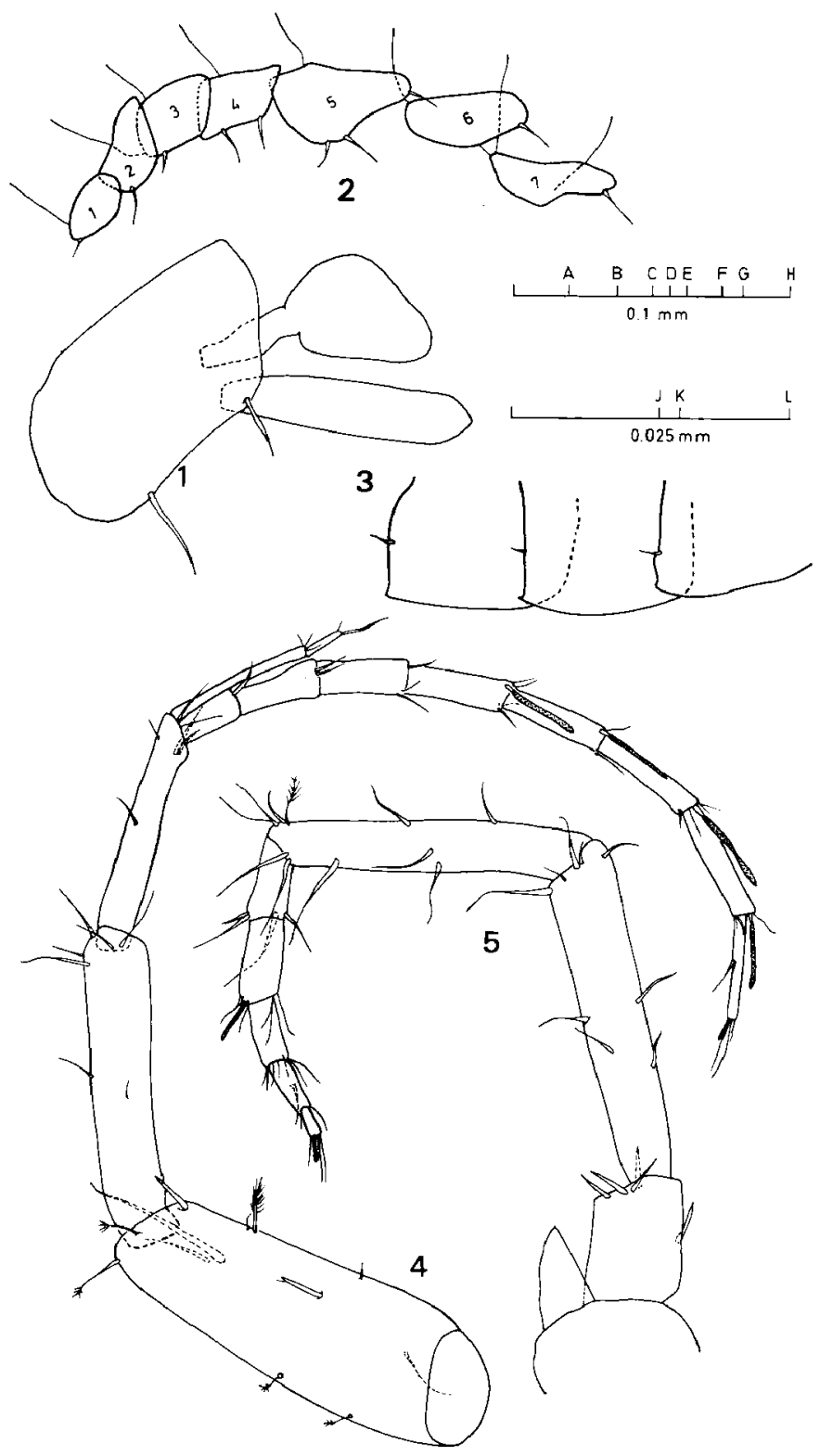

Bogidiella thai sp. n., ९.

1 : plaque coxale du péréionite 4 avec oostégite et branchie coxale - 2 : plaques coxales 1-7 - $3:$ plaques épimérales 4 : antenne $1-5$ : antenne $2.1,4,5$ : echelle $\mathrm{D} ; 2,3: \mathbf{A}$. 
du lobe prolongeant son angle postero-distal ; ce lobe est pointu, recouvert de cils et muni de 2 soies préapicales. Le propode présente sa dilatation maximum à son milieu environ, et c'est dans la zone de l'angle - fort accusé - du bord palmaire que se placent les éléments les plus caractéristiques de l'armature du bord palmaire oblique : deux assez fortes épines bifides, avec une soie normale entre elles ; une petite épine est placée un peu plus distalement par rapport à ce groupe, et une courte soie s'insère un peu plus proximalement ; $i$ n'y a pas de fine denticulation du bord palmaire; index palmaire (sensu Ruffo, 1973) : 0,47 che? la \&. Dactyle avec deux petites « dents " au bord postérieur, des sétules s'insérant au voisinage immédiat de ces " dents ". Pas de limite distincte entre dactyle et griffe.

Gnathopode 2 (fig. 21). Carpe nettement plus allongé que pour gn. 1, à angle postero-distal simplement arrondi. Propode très légèrement moins robuste que pour gn. 1, présentant sa dilatation maximum à son milieu environ, et c'est toujours dans la zone de l'angle - fort accusé - du bord palmaire que se situent les éléments les plus caractéristiques de l'armature du bord palmaire oblique : deux épines assez fortes, bifides, et une assez longue soie qui s'insère tout près de la plus distale de ces deux épines ; une petite épine est située un peu plus distalement par rapport à ce groupe, et une très longue soie $s^{t}$ insère un peu plus proximalement : index palmaire, $₹: 0,45$. Dactyle et griffe comme pour le gnathopode 1 .

Péréiopodes 3.7 (fig. 22-23). Certains péréiopodes (surtout P5 et P7) manquant dans nos exemplaires, il n'est pas possible de présenter des données comparatives sur leur longueur. Organes lenticulaires absents.

Les pléopodes (fig. 24) sont dépourvus d'endopodite ; leur exopodite ne présente pas de dimorphisme sexuel. Il n'y a pas d'épine modifiée dans l'arrnature du 2 ème pléopode $\sigma$. Basipodite avec 2 retinacula medio-distaux. Les trois segments de l'exopodite sont nettement plus courts depuis le $1^{\text {er }}$ jusqu'au 3ème, chaque segment offrant apicalement insertion à 2 longues soies recourbees et pennées.

Uropodes 1 et 2 ne présentant pas de dimorphisme sexuel, et sans épine modifiée dans l'armature de leurs rami. U1 (fig. 25) : rami subégaux, nettement plus courts que le pédoncule qui est long et assez gracile; pédoncule avec une épine insérée un peu plus basalement par rapport à la moitié de la longueur, et avec 2 épines aux angles distaux; 4 épines de tailles différentes à la partie distale de chaque ramus. U2 (fig. 26) : rami subégaux, approximativement de la même longueur que le pédoncule qui est court ; 2 épines distales sur le pédoncule, 4 épines de tailles différentes à la partie distale de chaque ramus.

Uropodes 3 (fig. 27). Pédoncule avec 3 épines distales; rami avec une armature d'épines bien fournie, une des épines apicales nettement plus longue que toutes les autres épines.

Telson (fig. 28-29) légèrement plus long que large, ayant une forme en quelque sorte polygonale. Emargination distale absente. Il y a 2 paires d'éperons ; une paire est subapicale, les deux éperons plus courts, relativement rapprochés l'un de l'autre; la zème paire s'insère latéralement à peu près au milieu du telson, et ces éperons - plus longs mais néanmoins nettement plus courts que le telson - sont largement séparés de ceux de la $1^{\text {ere }}$ paire. 1-2 soies sensorielles extrêmement fines s'insèrent un peu plus distalement par rapport aux éperons de la paire proximale.

Derivatio nominis. - Thaï est le nom du groupe ethnique principal de la Thaïlande, et de la langue du pays.

Considérations diverses. - Bogidiella thai sp. n. appartient au sous-genre Bogidiella s.str. par tous les caractères considérés dans les récentes révisions du genre (Stock 1981 ; Karaman 1982) comme distinctifs pour ce sous-genre. En effet, les pléopodes sont dépourvus d'endopodite, leur exopodite ne présente aucun dimorphisme sexuel, il n'y a pas de dimorphisme sexuel dans les uropodes 1 et 2 , dont les rami sont baculiformes et à épines non modifiées.

Il y a actuellement 21 taxa décrits considérés comme appartenant certainement ou éventuelle. ment au sous-genre Bogidiella (Coineau \& Stock 1986 ; Stock \& Notenboom, sous presse). Parmi les caractères les plus intéressants de la nouvelle espèce, permettant - en diverses combinaisons - de la distinguer de ces taxa, nous pouvons mentionner : flagelle accessoire de Al de 2 segments, le $1^{\text {er }}$ quatre fois plus long que le 2ème ; pars molaris des mandibules très fortement développée ; épines apicales du lobe externe de mx 1 pourvues de nombreux forts denticules; présence à l'apex du lobe interne du 


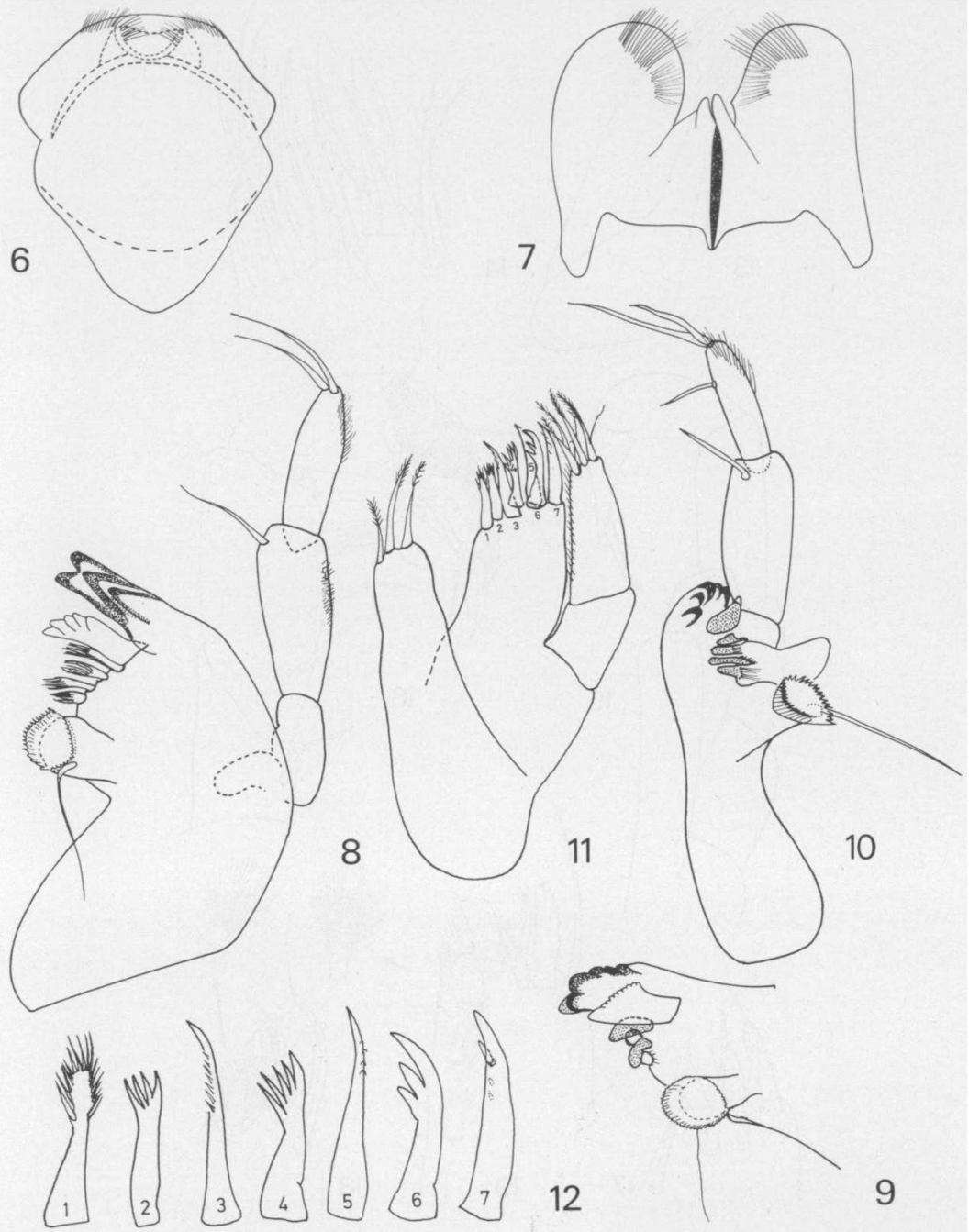

Bugidiella thai sp. n. 6 : labrum ( $)-7$ : labium $(\circ)-8:$ mandibule gauche $(8)-9:$ partie apicale du corps de la mandi. bule droite $(q)-10:$ mandibule droite $(\sigma)-11$ : maxille 1 gauche $(Q$; les éléments apicaux du lobe externe sont numérotés comme dans fig. 12) -12 : les 7 éléments apicaux du lobe exteme de mx $1(\$)$ à fort grossissement et numérotés comme dans fig. 11. 6,7 : échelle $\mathrm{E} ; 8,9,11: \mathrm{G} ; 10: \mathrm{F} ; 12: \mathrm{J}$. 


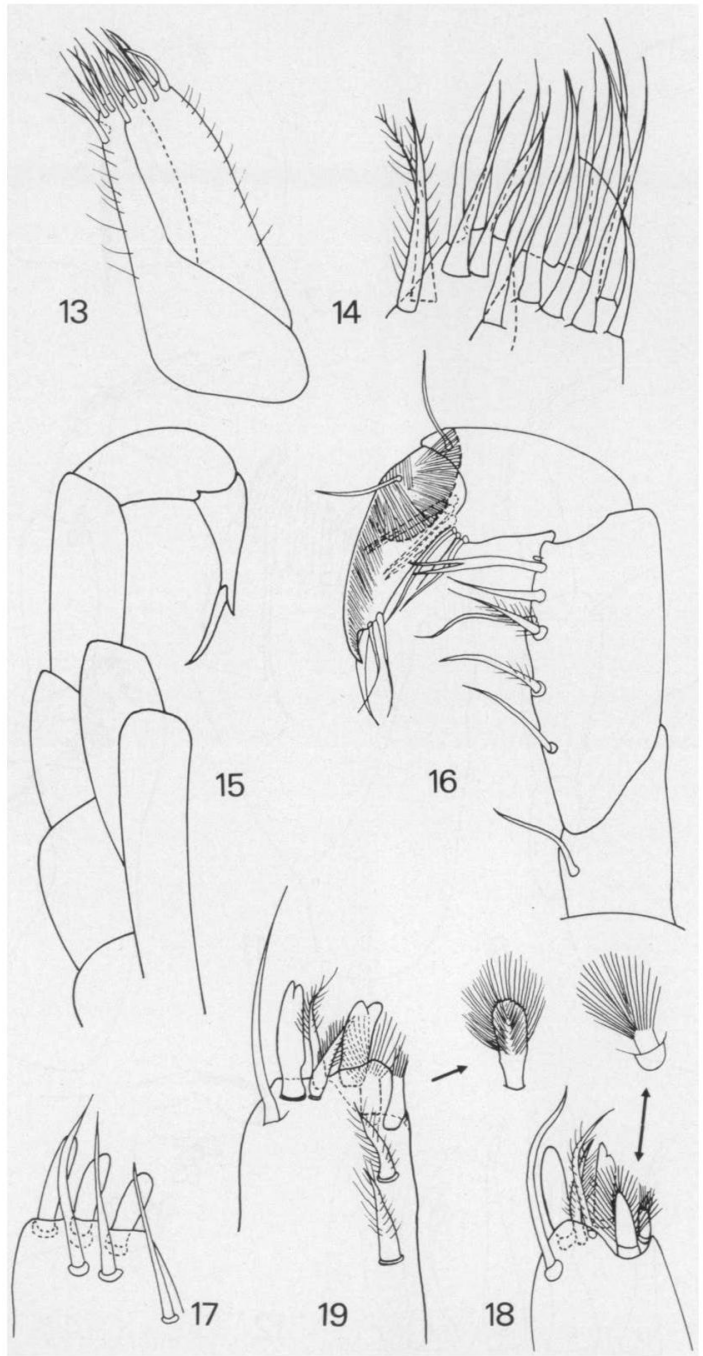

Bogidiella thai sp. n. 13 : maxille $2(\%)-14$ : apex des deux lobes de $m x 2(\ell)-15:$ maxillipède $(\varnothing)$, représentation simplifiée des différentes parties - 16: palpe du maxillipède $(0)-17$ et $18:$ partie apicale du lobe externe (17) et du lobe interne (18) du maxillipède $(\sigma)-19$ : partie apicale du lobe interne du maxillipede ( $Q$ ). Les flèches indiquent un autre aspect de l'une des fortes epines pennées. $13,15:$ échelle $\mathrm{G} ; 14,19: \mathrm{J} ; 16: \mathrm{H} ; 17,18: \mathrm{K}$. 


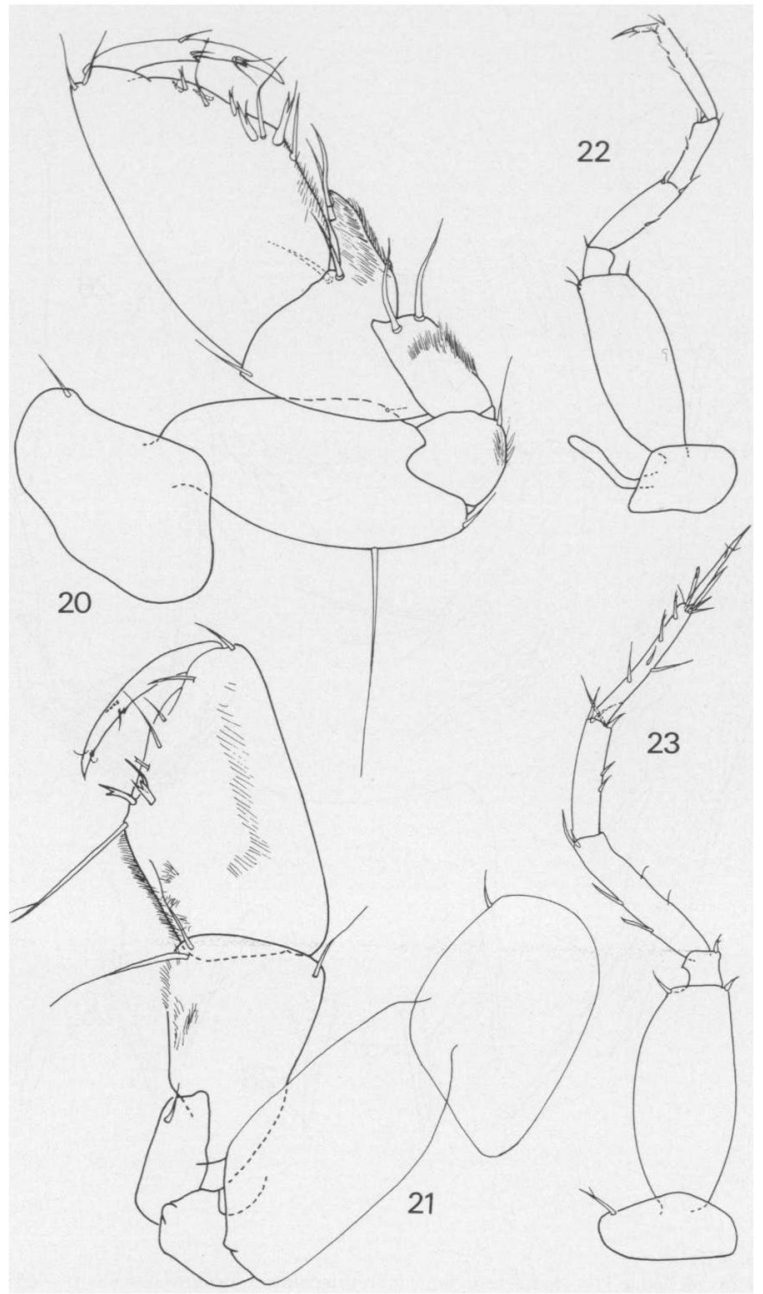

Bogidiella thai sp. n., ९. $20:$ gnathopode $1-21$ : gnathopode $2-22$ et 23 : péréiopodes 3 et $6.20,21:$ échelle D ; $22,23:$ A. 


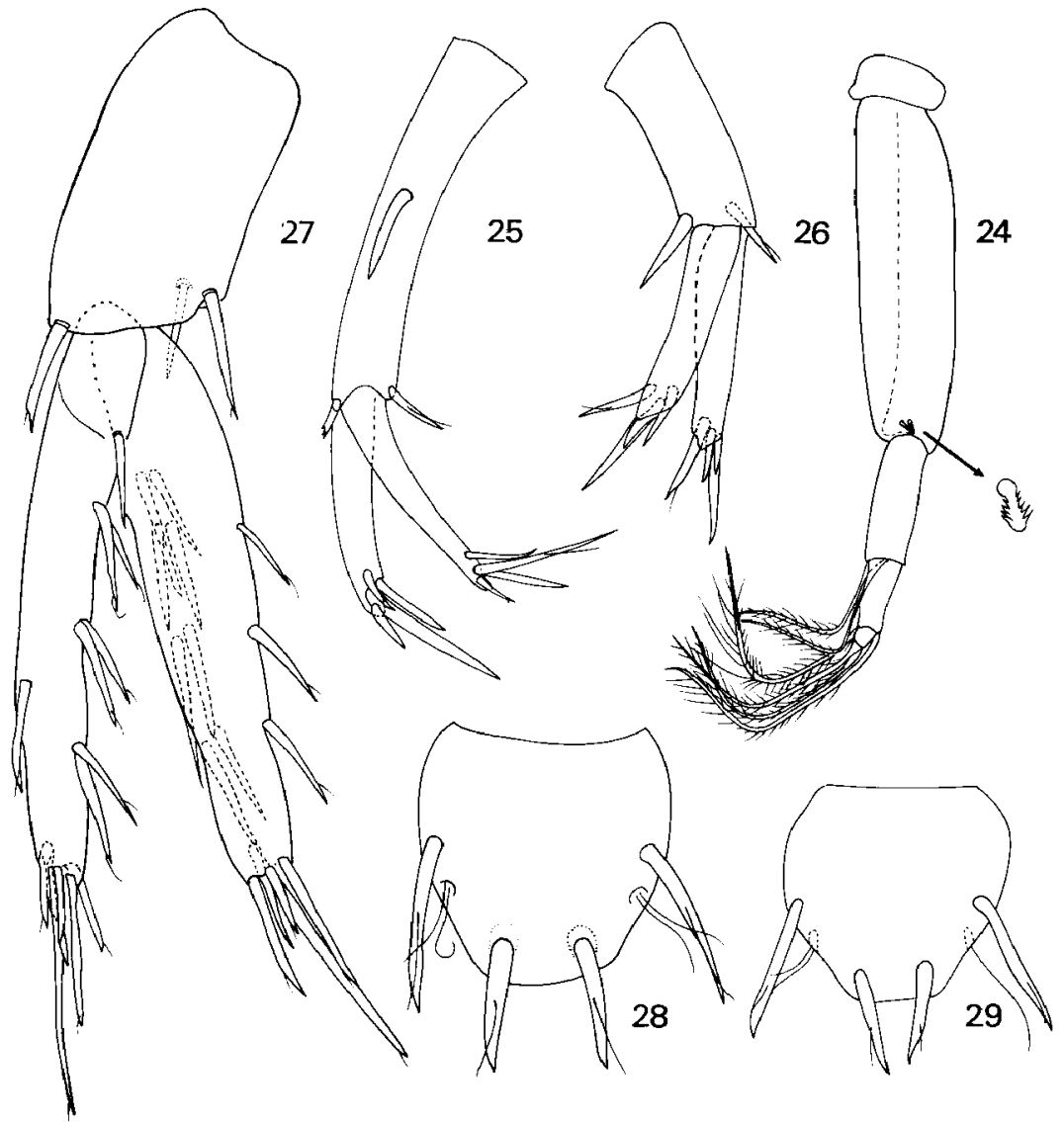

Be sidiella thai sp. n. 24 : pléopode 1 ( $\sigma$; la flèche indique un retinaculum à fort grossissement) - $25:$ uropode $1(\sigma)-26$ : uropode $2(\sigma)-27:$ uropode $3(9)-28$ et $29:$ telson $(28: \odot ; 29: \sigma)$. 24 : échelle B; $25-26:$ C ; $27:$ D $; 28-29:$ F 
mxp. d'une armature de 7 éléments, à l'angle interne se trouvant deux solides épines caractéristiquement foumies de longs cils ; apex du lobe externe du mxp. avec 3 épines à apex fort arrondi; extrémité du dactyle du palpe du mxp. prolongée en "bec "surplombant la racine de la griffe ; angle accentué du bord palmaire du propode de gn. 1 et surtout de gn. 2 , et concentration des épines fort peu nombreuses du bord palmaire en proximité de cet angle; absence des organes lenticulaires des péréiopodes ; proportions des segments de $\mathrm{U} 1$ et $\mathrm{U} 2$; nombre relativement élevé d'épines maginales des rami de U3 ; telson légèrement polygonal, totalement dépourvu d'émargination apicale et avec une disposition assez particulière de ses deux paires d'éperons.

Les espèces de bogidiellides géographiquement les plus proches de Bogidiella thai sp. n. sont : Bollegidia sootai (Coineau \& Rao 1972), espèce interstitielle marine décrite des Iles Andaman et Nicobar (Golfe du Bengale) et retrouvée en Malaisie (Ruffo 1985) ; et Bogidiella (sg. Medigidiella) sarawacensis Stock, 1983, espèce cavernicole de Sarawak, Borneo. Compte tenu de la vaste distribution des bogidiellides et du genre Bogidiella en particulier (Stock 1981), la découverte d'une nouvelle espèce en Thaïlande ne peut pas surprendre.

\section{Remerciements}

Nous remercions le $\mathrm{D}^{\mathbf{r}} \mathbf{L}$. Deharveng, de l'Université de Toulouse, pour nous avoir confié cet intéressant matériel.

\section{Travaux clites}

Coineau (N.) \& Chandrasekhara Rao (G.). 1972. - Isopodes et Amphipodes des sables intertidaux des Iles Andaman et Nicobar (Golfe du Bengale). Vie Milieu [ser. A], 23(1-A): 65-100.

Coineau (N.) \& Stock (J.H.). 1986, - Amphipoda : Bogidiellidae. in Botosaneanu (L.): Stygofauna Murtit : 574-580. Brill/Backhuys, Leiden

Karaman (G.S.). 1982. - Critical remarks to the recent revisions of Bugidielia-group of genera with study of some taxa (Fam. Gammaridae). Poljopr. Sem. (Titograd), 28(3-4): $31-57$.

Ruffo(S). 1973 - Contributo alla revisione det genere Bogidiella Hertzog (Crustacea Amphipoda, Gammaridae). Boll. Ist. Ent. Univ. Bologna, $31: 49-76$.

Ruffo (S.). 1985. - Nuovi Anfipodi mesopsammici delle Isole Andamane (Crust. Amphipoda). Boll. Mis. Civ. Stor. rat. Verona, $10: 485-509$.

Stock (J.H.). 1981. - The taxonomy and zoogeography of the family Bogidielfidae (Crustacea, Amphipoda). With emphasis on the West-Indian taxa. Bijdr. Dierk, 51(2): 345-374.

Stock (J.H.) 1983 . - Discovery of a Bogidiellid Amphipod Crus tacean in inland waters of the East lndian Archipelago: Bogidiella (Medigidiella). sarawacensis n. sp. Crustaceana, 44(2): 148.204.

Stock (J.H.) \& Natenboom (J.). - Five new bogidiellid Amphipoda Erom Spain - the first freshwater records in the Iberian Peninsula. Hydrobiologia (sous presse). 